\title{
Multiplexing using isothermal Recombinase Polymerase
}

\section{Amplification (RPA).}

RPA is an isothermal nucleic acid amplification technology which works with a single primer pair per target, similar to PCR. A number of alternative isothermal methods, including LAMP, require more than two primers per target. As only two primers are required for RPA, multiplexing is relatively simple.

Multiplexing is the simultaneous amplification of more than one target in a single reaction. The primers used for the amplification of each of the multiplexed targets have to be tested and potentially optimised for compatibility.

\section{Primer compatibility and concentration ratios}

A good strategy for identifying good primers for multiplex reactions is to first define a number of good candidate primer pairs for each target individually. Subsequently, the candidate pairs for each target are successively combined with the candidate pairs of the other targets in the final multiplex format. Having defined the best combination, differences in the relative performance of the primer pairs for the various targets can be adjusted by changing the ratios of the amount of primers used in the reaction.

\section{Different amplification rates}

The rate of product formation in different RPA assays can vary. Under circumstances in which target amplicons must be co-amplified, and their expected target numbers in a sample are similar, then identical amplification rates of the two targets may be optimal. However, in other circumstances it may be the case that one target will always be in significant excess over the other, and sensitivity of the less abundant target must be maintained. RPA, in contrast to PCR, offers advantages in this case as it is possible, by choice of primers, to develop a multiplex system in which the amplification rate of the less abundant target is faster than the more abundant species. This can prevent rapid amplification of the abundant target suppressing the detection of the less abundant target as it will only reach detectable levels later during the reaction. This gives the less abundant target ample time to reach detection before reagents are depleted. This flexibility is very useful when detection of a rare event is desired in a background of an abundant target which is to be used as an internal control.

\section{Example Multiplex RPA assays}

Duplex RPA assays, with a DNA target and reaction internal control working in parallel can be seen in both our lateral flow and fluorescence detection solutions for Salmonella enterica DNA target (see figures below).

Key for Figures: Sal+, reactions with 100cp of template DNA added; Sal-, reactions containing no template DNA (negative control); RPA IC, internal control.

Find out more about RPA technology here: www.twistdx.co.uk/en/rpa

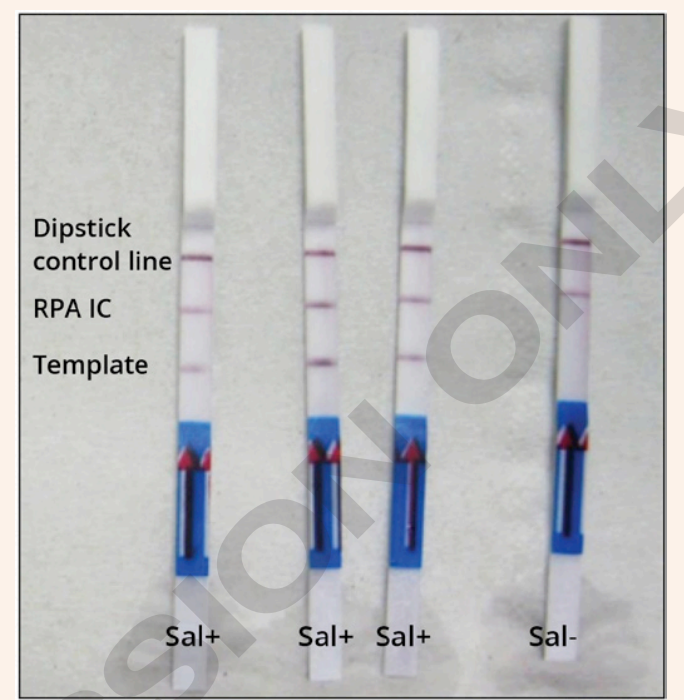

FIGURE 1. Lateral flow detection of S. enterica DNA amplification using the TwistFlow ${ }^{\circledR}$ Salmonella kit (www. twistdx.co.uk/en/products/ product/twistflow-salmonella) with Milenia HybriDetect 2 dipsticks (https://www.twistdx. co.uk/en/products/product/mileniahybridtech-2).

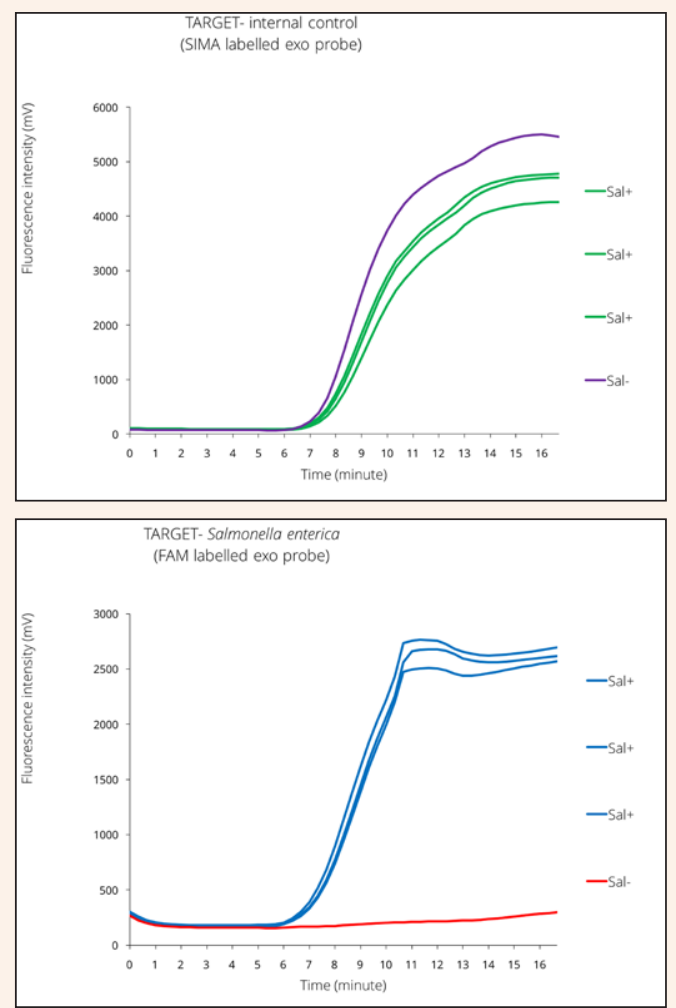

FIGURE 2. Figure 2. Real-time fluorescence detection of S. enterica DNA amplification using TwistGlow ${ }^{\circledR}$ Salmonella kit(www.twistdx.co.uk/en/ products/product/twistglow-salmonella). 